\title{
LINK BETWEEN HOSOYA INDEX AND FIBONACCI NUMBERS
}

\author{
HACÈNE BELBACHIR AND HAKIM HARIK
}

Received 09 November, 2014

\begin{abstract}
Let $G$ be a graph and $Z(G)$ be its Hosoya index. We show how the Hosoya index can be a good tool to establish some new identities involving Fibonacci numbers. This permits to extend Hillard and Windfeldt work.
\end{abstract}

2010 Mathematics Subject Classification: 05A19; 05C30; 11B39

Keywords: Fibonacci numbers, matching, Hosoya index, paths

\section{INTRODUCTION}

We denote by $G=(V(G), E(G))$ a simple undirected graph, $V(G)$ is the set of its vertices and $E(G)$ is the set of its edges. The order of $G$ is $|V(G)|$ and the size of $G$ is $|E(G)|$. For a vertex $v$ of $G, N(v)$ is the set of vertices adjacent to $v, \operatorname{deg}(v):=|N(v)|$ is the degree of $v ; \operatorname{Link}(v)$ is the set of edges incident to $v$. An edge $\{u, v\}$ of $G$ is denoted $u v$. A path $P_{n}$, from a vertex $v_{1}$ to a vertex $v_{n}$, $n \geq 2$, is a sequence of vertices $v_{1}, \ldots, v_{n}$ and edges $v_{i} v_{i+1}$, for $i=1, \ldots, n-1$; for simplicity we denote it by $v_{1} \cdots v_{n}$. We extend the definition of $P_{n}$ to $n=0$ and $n=1$ by setting $P_{0}$ is empty and $P_{1}$ is a single vertex, we add the convention that $P_{n} P_{0}=P_{0} P_{n}=P_{n}$ for all $n \geq 1$.

The graph $G-v$ is obtained from $G$ by removing the vertex $v$ and all edges of $G$ which are incident to $v$. For an edge $e$ of $G$, we denote by $G-e$ the graph obtained from $G$ by removing $e$. The contraction of a graph $G$, associated to an edge $e$, is the graph $G / e$ obtained by removing $e$ and identifying the vertices $u$ and $v$ incident to $e$ and replacing them by a single vertex $v^{\prime}$ where any edges incident to $u$ or $v$ are redirected to $v^{\prime}$. We then say that we contract in $G$ the adjacent vertices $u, v$ into the vertex $v^{\prime}$.

The well-known Fibonacci sequence $\left\{F_{n}\right\}$ is defined as $F_{0}=0, F_{1}=1$, and $F_{n}=$ $F_{n-1}+F_{n-2}$, for $n \geq 2$. The Fibonacci numbers are connected to the element of Pascal's triangle using the following identity

$$
F_{n+1}=\sum_{k=0}^{n}\left(\begin{array}{c}
n-k \\
k
\end{array}\right) \text {. }
$$


For some results and properties related to Fibonacci numbers, see for instance [1]. Many fields widely applies this sequence, particularly in physics and chemistry [10].

A matching $M$ of a graph $G$ is a subset of $E(G)$ such that no two edges in $M$ share a vertex in $G$. A matching of $G$ is also called an independent edge set of $G$. A $k$-matching of a graph $G$ is a matching of $G$ of cardinal $k$, it is then an independent edge set of $G$ of cardinal $k$. We denote by $m(G, k)$ the number of $k$-matchings of $G$ with the convention $m(G, 0)=1$. Note that $m(G, 1)=|E(G)|$ and when $k>n / 2$, $m(G, k)=0$.

The Hosoya index of a graph $G$, denoted by $Z(G)$, is an index introduced by Hosoya [9] as follows.

$$
Z(G)=\sum_{k=0}^{\lfloor n / 2\rfloor} m(G, k),
$$

where $n=|V(G)|,\lfloor n / 2\rfloor$ stands for the integer part of $n / 2$. This index has several applications in molecular chemistry such as boiling point, entropy or heat of vaporization. The literature includes many papers dealing the Hosoya index [2, 3, 6].

\section{Preliminary Results}

Before proving our main results, we first list the following results. From the definition of the Hosoya index, it is not difficult to deduce the following Lemma.

Lemma 1 ([7]). Let $G$ be a graph, we have

(1) If $u v \in E(G)$, then $Z(G)=Z(G-u v)+Z(G-\{u, v\})$.

(2) If $v \in V(G)$, then $Z(G)=Z(G-v)+\sum_{w \in N_{G}(v)} Z(G-\{w, v\})$.

(3) If $G_{1}, G_{2}, \ldots, G_{t}$ are the components of $G$ then $Z(G)=\prod_{k=1}^{t} Z\left(G_{k}\right)$.

Lemma 1 allows us to compute $Z(G)$ for any graph recursively.

The following theorem gives a relation between Hosoya index and Fibonacci number (see [5], [7]).

Theorem 1. Let $P_{n}$ be a path on $n$ vertices, then $Z\left(P_{n}\right)=F_{n+1}$.

\section{MAin RESUlts}

In this section, inspired by [4], we give another proofs of well-known identities (see Lemmas 2 and 3). Our goal is to prove the formula of Lemma 3 via Theorem 1. For this we give a direct proof of Lemma 2. We also establish two new identities, given in Theorems 2 and 3.

The following identity shows the relation between independent edge subsets in $P_{r_{1}+r_{2}}, P_{r_{1}}$ and $P_{r_{2}}$ for $r_{1}$ and $r_{2}$ two non-negative integers.

Lemma 2. Let $r_{1}, r_{2}$ be two non-negative integers, then

$$
Z\left(P_{r_{1}+r_{2}}\right)=Z\left(P_{r_{1}}\right) Z\left(P_{r_{2}}\right)+Z\left(P_{r_{1}-1}\right) Z\left(P_{r_{2}-1}\right) \text {. }
$$


Proof. Let $v_{1} \cdots v_{r_{1}+r_{2}}$ be a path $P_{r_{1}+r_{2}}$ partitioned into two paths $P_{r_{1}}$ represented by a sequence of vertices $v_{1} \cdots v_{r_{1}}$ and $P_{r_{2}}$ represented by a sequence of vertices $v_{r_{1}+1} \cdots v_{r_{1}+r_{2}}$. The vertices $v_{r_{1}}$ and $v_{r_{1}+1}$ share the same edge in $P_{r_{1}+r_{2}}$. The Hosoya number $Z\left(P_{r_{1}+r_{2}}\right)$ of the path $P_{r_{1}+r_{2}}$ represents the number of independent edge subsets between the vertices of $P_{r_{1}+r_{2}}$, this index can be written as $Z\left(P_{r_{1}+r_{2}}\right)=|M|+\left|M^{\prime}\right|$, where:

- $M$ is the set of independent edge subsets of $P_{r_{1}+r_{2}}$ such that the edge $v_{r_{1}} v_{r_{1}+1}$ does not belong to any independent edge subset of $M$, that means all independent edge subsets of $M$ are in $P_{r_{1}}$ and $P_{r_{2}}$, so $|M|=Z\left(P_{r_{1}}\right) Z\left(P_{r_{2}}\right)$.

- $M^{\prime}$ is the set of independent edge subsets of $P_{r_{1}+r_{2}}$ such that the edge $v_{r_{1}} v_{r_{1}+1}$ belongs to all independent edge subsets of $M^{\prime}$, that means the others independent edges of every subset of $M^{\prime}$ are in $P_{r_{1}-1}$ and $P_{r_{2}-1}$, so $|M|=Z\left(P_{r_{1}-1}\right) Z\left(P_{r_{2}-1}\right)$.

Lemma 3. Let $k, n$ be two integers such that $1 \leq k \leq n$. Then

$$
F_{n+1}=F_{k} F_{n-k}+F_{k+1} F_{n-k+1} .
$$

Proof. Consider a path $P_{n}=v_{1} \cdots v_{n}$ on $n$ vertices. We set $r_{1}:=k, r_{2}:=n-k$, and use Theorem 1 and Lemma 2.

We introduce a new identity of Fibonacci numbers which generalize identities of Fibonacci numbers given in [8].

For every integer $s \geq 2$, let $\Omega_{s}$ be the set of $\varepsilon:=\left(\varepsilon_{1}, \varepsilon_{2}, \ldots, \varepsilon_{s}\right)$ with $\varepsilon_{i} \in\{-1,0,1\}$ $(1 \leq i \leq s)$ such that :

(1) The number of $\varepsilon_{i}=0$ is even. Let $2 h(\varepsilon)$ this number.

(2) If $2 h(\varepsilon)=0$, then $\varepsilon_{i}=1$ for all $i$.

(3) If $2 h(\varepsilon) \neq 0$, let $L_{\varepsilon}:=\left\{s_{1}, s_{2}, \ldots, s_{2 h(\varepsilon)}: s_{1}<s_{2}<\ldots<s_{2 h(\varepsilon)}\right.$ and $\varepsilon_{s_{i}}=$ 0 for all $i \in\{1,2, \ldots, 2 h(\varepsilon)\}\}$.

For all $l \in L_{\varepsilon}$, we have :

- $l$ is even $\Longrightarrow \varepsilon_{i}=1$ for all $s_{l}<i<s_{l+1}$;

- $l$ is odd $\Longrightarrow \varepsilon_{i}=-1$ for all $s_{l}<i<s_{l+1}$;

- $\varepsilon_{i}=1$ for all $i<s_{1}$ or $i>s_{2 h(\varepsilon)}$.

For example,

$$
\begin{gathered}
\Omega_{2}=\{(1,1),(0,0)\}, \\
\Omega_{3}=\{(1,1,1),(1,0,0),(0,0,1),(0,-1,0)\}, \\
\Omega_{4}=\{(1,1,1,1),(0,0,1,1),(1,0,0,1),(1,1,0,0),(0,0,0,0),(0,-1,0,1), \\
(1,0,-1,0),(0,-1,-1,0)\} .
\end{gathered}
$$

- The lines of the following table represent the elements of $\Omega_{5}$, 


$$
\left|\begin{array}{rrrrr||rrrrr}
1 & 1 & 1 & 1 & 1 \\
0 & 0 & 1 & 1 & 1 \\
1 & 0 & 0 & 1 & 1 & 0 & -1 & -1 & 0 & 1 \\
1 & 0 & -1 & -1 & 0 \\
0 & -1 & -1 & -1 & 0 \\
1 & 1 & 0 & 0 & 1 & 0 & 0 & 0 & 0 & 1 \\
1 & 1 & 1 & 0 & 0 & 0 & 0 & 1 & 0 & 0 \\
0 & -1 & 0 & 1 & 1 & 1 & 0 & 0 & 0 & 0 \\
1 & 0 & -1 & 0 & 1 & 0 & -1 & 0 & 0 & 0 \\
1 & 1 & 0 & -1 & 0 & 0 & 0 & 0 & -1 & 0
\end{array}\right|
$$

- The lines of the following table represent the elements of $\Omega_{6}$,

$$
\left|\begin{array}{llllll||llllll}
1 & 1 & 1 & 1 & 1 & 1 \\
0 & 0 & 1 & 1 & 1 & 1 & 0 & 0 & 0 & 0 & 1 & 1 \\
1 & 0 & 0 & 1 & 1 & 1 & 0 & 1 & 0 & 0 & 1 \\
0 & 0 & 1 & 1 & 0 & 0 \\
1 & 1 & 0 & 0 & 1 & 1 & 0 & 0 & 0 & 0 & 1 \\
1 & 1 & 1 & 0 & 0 & 1 & 0 & 0 & 0 & 1 & 0 & 0 \\
1 & 1 & 1 & 1 & 0 & 0 & 1 & 1 & 0 & 0 & 0 & 0 \\
0 & -1 & 0 & 1 & 1 & 1 & 0 & -1 & 0 & 0 & 0 & 1 \\
1 & 0 & -1 & 0 & 1 & 1 & 0 & -1 & 0 & 1 & 0 & 0 \\
1 & 1 & 0 & -1 & 0 & 1 & 1 & 0 & -1 & 0 & 0 & 0 \\
1 & 1 & 1 & 0 & -1 & 0 & 0 & 0 & 0 & -1 & 0 \\
0 & -1 & -1 & 0 & 1 & 1 & 0 & 0 & 1 & 0 & -1 & 0 \\
1 & 0 & -1 & -1 & 0 & 1 & 0 & 0 & 0 & -1 & 0 & 1 \\
1 & 1 & 0 & -1 & -1 & 0 & 0 & -1 & 0 & 0 & -1 & 0 \\
0 & -1 & -1 & -1 & 0 & 1 & 0 & 0 & -1 & -1 & 0 \\
1 & 0 & -1 & -1 & -1 & 0 & 0 & -1 & -1 & 0 & 0 & 0 \\
0 & -1 & -1 & -1 & -1 & 0 & 0 & 0 & 0 & 0 & 0 & 0
\end{array}\right|
$$

Theorem 2. For any positive integers $r_{i}(1 \leq i \leq s)$ and any integer $s \geq 2$, we have

$$
F_{r_{1}+r_{2}+\cdots+r_{s}+1}=\sum_{\left(\varepsilon_{1}, \varepsilon_{2}, \ldots, \varepsilon_{s}\right) \in \Omega_{s}} \prod_{i=1}^{s} F_{r_{i}+\varepsilon_{i}},
$$

Proof. Let $P_{r_{1}+r_{2}+\cdots+r_{s}}$ be a path with $r_{1}+r_{2}+\cdots+r_{s}$ vertices. We subdivide $P_{r_{1}+r_{2}+\cdots+r_{s}}$ in consecutive blocs of paths $P_{r_{i}}$ with $r_{i}$ vertices $(1 \leq i \leq s)$, see Figure 1.

In one hand side, by Theorem 1, we have $Z\left(P_{r_{1}+\cdots+r_{s}}\right)=F_{r_{1}+\cdots+r_{s}+1}$. In the other hand $Z\left(P_{r_{1}+r_{2}+\cdots+r_{s}}\right)$ is the number of independent edge subsets in $P_{r_{1}+r_{2}+\cdots+r_{s}}$. So, $Z\left(P_{r_{1}+r_{2}+\cdots+r_{s}}\right)=\sum_{i=0}^{s-1}\left|M_{k}\right|$ where $M_{k}$ is the set of independent edge subsets $I$ in $P_{r_{1}}+r_{2}+\cdots+r_{s}$ such that it exists exactly $k$ edges between blocs of paths $P_{r_{i}}(1 \leq i \leq s)$ which belong to $I$. 


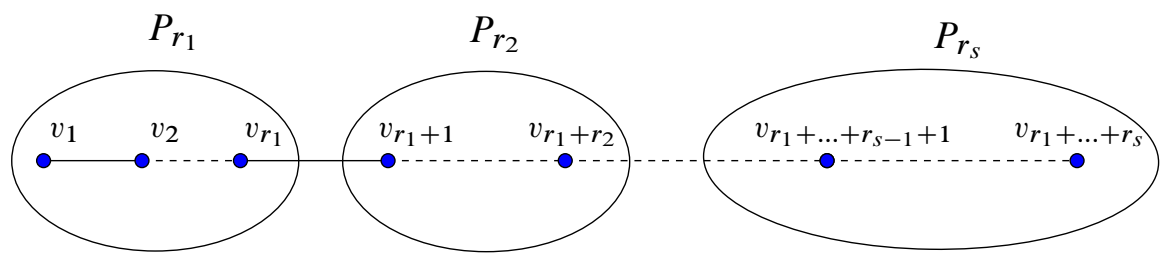

FIGURE 1. Path $P_{r_{1}+r_{2}+\ldots+r_{s}}$ divided in consecutive blocs of paths $P_{r_{i}}$ with $r_{i}$ vertices $(1 \leq i \leq s)$.

$M_{0}$ is the set of independent edge subsets $I$ in $P_{r_{1}+r_{2}+\cdots+r_{s}}$ such that doesn't contain any edge between blocs of paths $P_{r_{i}}(1 \leq i \leq s)$, so all these independent edge subsets are in blocs $P_{r_{i}}(1 \leq i \leq s)$. Hence, $\left|M_{0}\right|=\prod_{i=1}^{s} F_{r_{i}+1}$.

$M_{1}$ is the set of independent edge subsets $I$ in $P_{r_{1}+r_{2}+\cdots+r_{s}}$ such that it exists only one edge between blocs of paths $P_{r_{i}}(1 \leq i \leq s)$ which belong to $I$. Let $H$ be a subset of $M_{1}$ containing the edge $v_{r_{1}+\cdots+r_{k}} v_{r_{1}+\cdots+r_{k}+1}(1 \leq k \leq s-1)$ in all of its independent edge subsets. We contract the adjacent vertices $v_{r_{1}+\cdots+r_{k}}, v_{r_{1}}+\cdots+r_{k}+1$ in $P_{r_{1}+\cdots+r_{s}}$ into one vertex $v^{\prime}$ and $P_{r_{1}+\cdots+r_{s}-1}$ is a new path after contraction composed of consecutive blocs of paths $P_{r_{1}}, P_{r_{2}}, \ldots, P_{r_{k-1}}, P_{r_{k}-1}, v^{\prime}, P_{r_{k+1}-1}, P_{r_{k+2}}, \ldots$, $P_{r_{s}}$. A path $P_{r_{1}+\cdots+r_{s}-1}$ does not contain any edge between blocs which belong to independent edge subsets of $H$, so $|H|=F_{r_{1}+1} \times F_{r_{2}+1} \times \cdots \times F_{r_{k-1}+1} \times F_{r_{k}} \times$ $F_{2} \times F_{r_{k+1}} \times F_{r_{k+2}+1} \times \cdots \times F_{r_{s}+1}$. Thus, $\left|M_{1}\right|=\sum_{\left(\varepsilon_{1}, \varepsilon_{2}, \ldots, \varepsilon_{s}\right) \in \Delta_{1}} \prod_{i=1}^{s} F_{r_{i}+\varepsilon_{i}}$ where $\Delta_{1}$ is the set of $\left(\varepsilon_{1}, \varepsilon_{2}, \ldots, \varepsilon_{s}\right)$ such that for $1 \leq i \leq s, \varepsilon_{i} \in\{0,1\}$ and $\varepsilon_{1} \varepsilon_{2} \cdots \varepsilon_{s}$ forms a sequence such that there is only one pair of zeros and this pair is of the form $\left(\varepsilon_{l}, \varepsilon_{l+1}\right)$.

$M_{2}$ is the set of independent edge subsets $I$ in $P_{r_{1}+r_{2}+\cdots+r_{s}}$ such that it exists exactly two edges between blocs of paths $P_{r_{i}}(1 \leq i \leq s)$ which belong to $I$. As for computing of $\left|M_{1}\right|$ and using the contraction method for the two edges between blocs of paths $P_{r_{i}}(1 \leq i \leq s)$, we have $\left|M_{2}\right|=\sum_{\left(\varepsilon_{1}, \varepsilon_{2}, \ldots, \varepsilon_{s}\right) \in \Delta_{2}} \prod_{i=1}^{s} F_{r_{i}+\varepsilon_{i}}$ where $\Delta_{2}$ is the set of $\left(\varepsilon_{1}, \varepsilon_{2}, \ldots, \varepsilon_{s}\right) \in \Omega_{s}$ such that for $1 \leq i \leq s, \varepsilon_{i} \in\{-1,0,1\}$ and $\varepsilon_{1} \varepsilon_{2} \cdots \varepsilon_{s}$ forms a sequence such that there is only one pair of zeros $\left\{\varepsilon_{i}, \varepsilon_{i+2}\right\}$ and $\varepsilon_{i+1}=-1$, or only two pairs of zeros $\left\{\varepsilon_{i}, \varepsilon_{i+1}\right\},\left\{\varepsilon_{i+1+k}, \varepsilon_{i+2+k}\right\}(1 \leq k$ and $i+k+2 \leq s)$ and $\varepsilon_{l}=1$ for all $l \in\{1,2, \ldots, i-1, i+2, i+3, \ldots, i+k, i+k+3, \ldots, s\}$.

For $M_{k}(3 \leq k \leq s-2)$, using the contraction method for $k$ edges between blocs of paths $P_{r_{i}}(1 \leq i \leq s)$, we have $\left|M_{k}\right|=\sum_{\left(\varepsilon_{1}, \varepsilon_{2}, \ldots, \varepsilon_{s}\right) \in \Delta_{k}} \prod_{i=1}^{s} F_{r_{i}+\varepsilon_{i}}$ where $\Delta_{k}=\left\{\left(\varepsilon_{1}, \varepsilon_{2}, \ldots, \varepsilon_{s}\right) \in \Omega_{s}:\right.$ for all $s_{i} \in L_{\varepsilon}(1 \leq i \leq 2 h(\varepsilon)), \sum_{l=1}^{h(\varepsilon)}\left(s_{2 l}-s_{2 l-1}\right)=$ $k\}$ which represents all sequences of $\Omega_{s}$ such that the sum of the difference of the position of each pair of 0 is equal to $k$.

We finish by $M_{s-1}$ which is the set of matchings $I$ in $P_{r_{1}+r_{2}+\cdots+r_{s}}$ such that it exists exactly $s-1$ edges between blocs of paths $P_{r_{i}}(1 \leq i \leq s)$ which belong to 
$I$. In this case, except the paths $P_{r_{1}}, P_{r_{s}}$ that lose one vertex after a contraction all others paths $P_{r_{i}}(2 \leq i \leq s-1)$ lose two vertices after contraction method. Thus, $\left|M_{s-1}\right|=F_{r_{1}} F_{r_{s}} \prod_{i=2}^{s-1} F_{r_{i}-1}$.

Note that $\left\{\Delta_{k}: 1 \leq k \leq s-1\right\}$ is a partition of $\Omega_{s}$. Hence, the identity (3.1) holds.

The following corollaries are the main results given by [8].

Corollary 1. For any non-negative integers $r$ and $t$, we have

$$
F_{r+t}=F_{r+1} F_{t}+F_{r} F_{t-1} .
$$

Proof. From Theorem 2 with $s=2$ and $\Omega_{2}=\{(1,1),(0,0)\}$, we obtain the following identity $F_{r_{1}+r_{2}+1}=F_{r_{1}+1} F_{r_{2}+1}+F_{r_{1}} F_{r_{2}}$. We put $r=r_{1}$ and $t=r_{2}+1$ and we conclude.

Corollary 2. For any non-negative integers $u$, $v$ and $w$, we have

$$
F_{u+v+w}=F_{u+1} F_{v+1} F_{w+1}+F_{u} F_{v} F_{w}-F_{u-1} F_{v-1} F_{w-1} .
$$

Proof. From Theorem 2 with $s=3$ and $\Omega_{3}=\{(1,1,1),(1,0,0),(0,0,1),(0,-1,0)\}$, we obtain the following identity $F_{r_{1}+r_{2}+r_{3}+1}=F_{r_{1}+1} F_{r_{2}+1} F_{r_{3}+1}+F_{r_{1}+1} F_{r_{2}} F_{r_{3}}+$ $F_{r_{1}} F_{r_{2}} F_{r_{3}+1}+F_{r_{1}} F_{r_{2}-1} F_{r_{3}}$. We put $u=r_{1}, v=r_{2}$ and $w=r_{3}+1$ and using $F_{t}=F_{t+1}-F_{t-1}$, we have :

$$
\begin{aligned}
F_{u+v+w} & =F_{u+1} F_{v+1} F_{w}+F_{u+1} F_{v} F_{w-1}+F_{u} F_{v} F_{w}+F_{u} F_{v-1} F_{w-1} \\
& =F_{u+1} F_{v+1}\left(F_{w+1}-F_{w-1}\right)+F_{u+1} F_{v} F_{w-1}+F_{u} F_{v} F_{w} \\
& +\left(F_{u+1}-F_{u-1}\right) F_{v-1} F_{w-1} \\
& =F_{u+1} F_{v+1} F_{w+1}-F_{u+1} F_{v+1} F_{w-1}+F_{u+1} F_{v} F_{w-1}+F_{u} F_{v} F_{w} \\
& +F_{u+1} F_{v-1} F_{w-1}-F_{u-1} F_{v-1} F_{w-1} \\
& =F_{u+1} F_{v+1} F_{w+1}+F_{u} F_{v} F_{w}-F_{u-1} F_{v-1} F_{w-1}+F_{u+1} F_{v-1} F_{w-1} \\
& +F_{u+1} F_{v} F_{w-1}-F_{u+1} F_{v+1} F_{w-1} \\
& =F_{u+1} F_{v+1} F_{w+1}+F_{u} F_{v} F_{w}-F_{u-1} F_{v-1} F_{w-1} \\
& +F_{u+1}\left(F_{v-1}+F_{v}-F_{v+1}\right) F_{w-1} \\
& =F_{u+1} F_{v+1} F_{w+1}+F_{u} F_{v} F_{w}-F_{u-1} F_{v-1} F_{w-1} .
\end{aligned}
$$

Corollary 3. For any non-negative integers $a, b, c$ and $d$, we have

$$
\begin{gathered}
F_{a+b+c+d+1} \\
=F_{a+1} F_{b+1} F_{c+1} F_{d+1}+F_{a} F_{b} F_{c} F_{d}+F_{a+1} F_{b} F_{c} F_{d+1}+F_{a+1} F_{b+1} \\
+F_{c} F_{d}+F_{a} F_{b-1} F_{c} F_{d+1}+F_{a} F_{b} F_{c+1} F_{d+1} \\
+F_{a+1} F_{b} F_{c-1} F_{d}+F_{a} F_{b-1} F_{c-1} F_{d} .
\end{gathered}
$$


Proof. From Theorem 2, with $s=4$ and $\Omega_{4}=\{(1,1,1,1),(0,0,1,1),(1,0,0,1)$, $(1,1,0,0),(0,0,0,0),(0,-1,0,1),(1,0,-1,0),(0,-1,-1,0)\}$, we obtain the identity.

The following theorem is another identity of Fibonacci number which gives an equivalent of Theorem 2.

Theorem 3. Let $s \geq 2$ be an integer. For any non-negative integer $r_{i}(1 \leq i \leq s)$, we have

$$
F_{\sum_{i=1}^{s} r_{i}+1}=F_{\sum_{i=1}^{s-1} r_{i}+1} F_{r_{s}+1}+\sum_{i=0}^{s-2}\left[\left(\prod_{j=1}^{i} F_{r_{s-j}-1}\right) F_{\sum_{j=1}^{s-i-2} r_{j}+1} F_{r_{s-i-1}} F_{r_{s}}\right] .
$$

Proof. As mentioned in Theorem 2, $F_{\sum_{i=1}^{s} r_{i}+1}=\sum_{\left(\varepsilon_{1}, \varepsilon_{2}, \ldots, \varepsilon_{s}\right) \in \Omega_{s}} \prod_{i=1}^{s} F_{r_{i}+\varepsilon_{i}}$. Then $F_{\sum_{i=1}^{s} r_{i}+1}=c_{1}+c_{0}$ where $c_{1}$ corresponds to the case $\varepsilon_{s}=1$ and $c_{0}$ to the case $\varepsilon_{s}=0$. That means to count $F_{\sum_{i=1}^{s} r_{i}+1}$ we have two cases.

Case 1. $\varepsilon_{s}=1$. Then for all s-uplet $\left(\varepsilon_{1}, \varepsilon_{2}, \ldots, \varepsilon_{s}\right)$ we obtain

$$
c_{1}=F_{r_{s}+1}\left(\sum_{\left(\varepsilon_{1}, \varepsilon_{2}, \ldots, \varepsilon_{s-1}\right) \in \Omega_{s-1}} \prod_{i=1}^{s-1} F_{r_{i}+\varepsilon_{i}}\right),
$$

so for $\varepsilon_{s}=1$ we have $c_{1}=F_{\sum_{i=1}^{s-1} r_{i}+1} F_{r_{s}+1}$.

Case 2. $\varepsilon_{s}=0$. Let $\varepsilon_{s-i-1}=0$ with $i$ the smallest integer $k, 0 \leq k \leq s-2$, such that $\varepsilon_{s-k-1}=0$. So for $1 \leq j \leq i$ we have $\varepsilon_{s-j}=-1$. Hence,

$$
\begin{aligned}
c_{0} & =\sum_{i=0}^{s-2}\left[\left(\prod_{j=1}^{i} F_{r_{s-j-1}}\right) F_{r_{s-i-1}} F_{r_{s}} \sum_{\left(\varepsilon_{1}, \varepsilon_{2}, \ldots, \varepsilon_{s-i-2}\right) \in \Omega_{s-i-2}} \prod_{j=1}^{s-i-2} F_{r_{j}+\varepsilon_{j}}\right] \\
& =\sum_{i=0}^{s-2}\left[\left(\prod_{j=1}^{i} F_{r_{s-j}-1}\right) F_{\sum_{j=1}^{s-i-2} r_{j}+1} F_{r_{s-i-1}} F_{r_{s}}\right] .
\end{aligned}
$$

As an immediate consequence of Theorem 3 we have :

Corollary 4. For any non-negative integers $s$ and $r$, we have

$$
F_{s r+1}=F_{r+1} F_{(s-1) r+1}+F_{r}^{2} \sum_{i=0}^{s-2} F_{r-1}^{i} F_{(s-i-2) r+1} .
$$

Proof. Use Theorem 3 with $r_{1}=r_{2}=\cdots=r_{s}=r$. 


\section{ACKNOWLEDGEMENT}

The authors wish to express their grateful thanks to the anonymous referee for her/his comments and suggestions towards revising this paper.

\section{REFERENCES}

[1] H. Belbachir and F. Bencherif, "Linear recurrent sequences and powers of a square matrix," Integers, vol. 6, pp. A12, 17, 2006.

[2] J. A. Bondy and U. S. R. Murty, "Graph theory with applications," North-Holland, 1976.

[3] O. Chan, I. Gutman, T. K. Lam, and R. Merris, "Algebraic connections between topological indices," J. Chem. Inform. Comput. Sci., vol. 38, pp. 62-65, 1998.

[4] H. Deng, "The largest Hosoya index of ( $n, n+1)$-graphs," Comput. Math. Appl., vol. 56, no. 10, pp. 2499-2506, 2008, doi: 10.1016/j.camwa.2008.05.020.

[5] I. Gutman, "Acyclic systems with extremal Hückel $\pi$-electron energy," Theoret. Chim. Acta, vol. 45, no. 1, pp. 79-87, 1977, doi: 10.1007/BF00552542.

[6] I. Gutman and S. J. Cyvin, "Hosoya index of fused molecules," MATCH Commun. Math. Comput. Chem., no. 23, pp. 89-94, 1988.

[7] I. Gutman and O. E. Polansky, Mathematical concepts in organic chemistry. Springer-Verlag, Berlin, 1986. doi: 10.1007/978-3-642-70982-1.

[8] C. J. Hillar and T. Windfeldt, "Fibonacci identities and graph colorings," Fibonacci Quart., vol. $46 / 47$, no. 3, pp. 220-224, 2008/09.

[9] H. Hosoya, "Topological index, a newly proposed quantity characterizing the topological nature of structural isomers of saturated hydrocarbons," Bull. Chem. Soc. Jap., vol. 44, no. 9, pp. 2332 2339, 1971.

[10] T. Koshy, Fibonacci and Lucas numbers with applications, ser. Pure and Applied Mathematics (New York). Wiley-Interscience, New York, 2001. doi: 10.1002/9781118033067.

Authors' addresses

Hacène Belbachir

USTHB, Faculty of Mathematics, RECITS Laboratory, DG-RSDT, Po. Box 32, El Alia, 16111, Algiers, Algeria

E-mail address: hbelbachir@usthb.dz or hacenebelbachiregmail.com

Hakim Harik

USTHB, Faculty of Mathematics, RECITS Laboratory, DG-RSDT, Po. Box 32, El Alia, 16111, Algiers, Algeria

Current address: CERIST, 5 Rue des frères Aissou, Ben Aknoun, Algiers, Algeria

E-mail address: hhakim@mail.cerist.dz, harik_hakim@yahoo.fr 